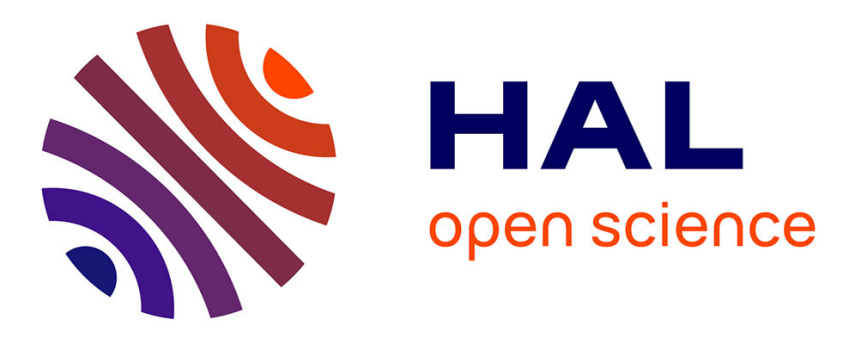

\title{
Hybrid Universal Generating Function for the Reliability Assessment of Multi-State Systems under Aleatory and Epistemic Uncertainties
}

\author{
Yan-Fu Li, Y. Ding, Enrico Zio
}

\section{- To cite this version:}

Yan-Fu Li, Y. Ding, Enrico Zio. Hybrid Universal Generating Function for the Reliability Assessment of Multi-State Systems under Aleatory and Epistemic Uncertainties. ESREL 2013, Sep 2013, Amsterdam, Netherlands. pp.1-8. hal-00911991

\section{HAL Id: hal-00911991}

\section{https://hal-centralesupelec.archives-ouvertes.fr/hal-00911991}

Submitted on 1 Dec 2013

HAL is a multi-disciplinary open access archive for the deposit and dissemination of scientific research documents, whether they are published or not. The documents may come from teaching and research institutions in France or abroad, or from public or private research centers.
L'archive ouverte pluridisciplinaire $\mathbf{H A L}$, est destinée au dépôt et à la diffusion de documents scientifiques de niveau recherche, publiés ou non, émanant des établissements d'enseignement et de recherche français ou étrangers, des laboratoires publics ou privés. 


\title{
Hybrid Universal Generating Function for the Reliability Assessment of Multi-State Systems under Aleatory and Epistemic Uncertainties
}

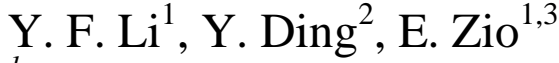 \\ ${ }^{1}$ Chair on Systems Science and the Energetic Challenge, European Foundation for New Energy-Electricite' \\ de France, at Ecole Centrale Paris-Supelec, Paris, France \\ ${ }^{2}$ Electrical Engineering Department, Technical University of Denmark, DK 2800, Denmark \\ ${ }^{3}$ Energy Department, Politecnico di Milano, Milano, Italy
}

\begin{abstract}
In this work, we extend the traditional universal generating function (UGF) approach for multistate system (MSS) reliability assessment to account for both aleatory and epistemic uncertainties. Firstly, a theoretical extension, named hybrid UGF (HUGF), is made to introduce the use of random fuzzy variables (RFVs) in the approach; secondly, the composition operator of HUGF is defined by considering simultaneously the probabilistic convolution and the fuzzy extension principle; finally, an efficient algorithm is designed to extract probability boxes (p-boxes) from the system HUGF, which allow quantifying different levels of imprecision in system reliability estimation. The HUGF approach is demonstrated on a numerical example.
\end{abstract}

\section{INTRODUCTION}

Multi-state system (MSS) modeling has been widely applied to resolve system reliability assessment problems (Natvig, 2011, Lisnianski and Levitin, 2003). Under this framework, the performance of each component is discretized into more than two exclusive states from perfect functioning to complete failure, and each state is characterized by a probability of occurrence. In general, MSS reliability assessment aims to derive the system availability $A(w)$ as the probability that the system performance $G_{S}$ is not less than the demand $w, A(w)=$ $P\left(G_{S Y S}-w \geq 0\right)$. $G_{S Y S}$ is determined by the MSS system structure, which is a function $\varphi(\cdot)$ of the $n$ component performance variables, $G_{S Y S}=$ $\varphi\left(G_{1}, \ldots, G_{i}, \ldots, G_{n}\right)$, where $G_{i}$ is the $i$-th component performance variable that takes values from the finite set $\left\{g_{i, 0}, \ldots, g_{i, j} \ldots, g_{i, M_{i}}\right\}$, where $g_{i, j}$ is the performance level of component $i$ at its state $j=0, \ldots, M_{i}$ and $M_{i}$ is the highest possible state of component $i$. Typically, $g_{i, 0}$ and $g_{i, M_{i}}$ represent the performance levels at complete failure and perfect functioning conditions, respectively.

Uncertainty is an unavoidable factor in MSS reliability assessment (Lisnianski and Levitin, 2003). Conventionally, the uncertain behavior of $G_{i}$ is described by its discrete probability distribution $P\left(G_{i}=g_{i, j}\right)$, such that $\sum_{j=0}^{M_{i}} P\left(G_{i}=g_{i, j}\right)=1$. The probability distribution is sufficient to describe the state randomness, i.e. uncertainty of objective and aleatory type due to the natural variability or stochasticity of the component behavior. Another type of uncertainty to account for is that due to the incomplete or imprecise knowledge about the component performance ( $\mathrm{Li}$ and Zio, 2012b, Singer, 1990, Lin et al., 2012, Wang et al., 2011, Cai, 1996, Chen, 1994). This type of uncertainty is often referred to as subjective and epistemic (Helton, 2004, Apostolakis, 1990). Recently, epistemic uncertainty in MSS models has been described by a fuzzy approach based on the universal generating function (UGF) paradigm (Ushakov, 1986), assuming that the state probabilities and/or the state performance can be fuzzy variables (FVs) (Ding et al., 2008, Ding et al., 2010). Interval values have also been used in ( $\mathrm{Li}$ et al., 2011) to represent the imprecision in both state probability and performance.

The issue of handling both types of uncertainties (aleatory and epistemic) has been an active research topic in the area of reliability and risk assessment (Ferson, 1996, Baudrit et al., 2006) since the 1990s. Aleatory, epistemic, mixed aleatory and epistemic uncertainties (due to partial variability and partial ignorance (Baraldi and Zio, 2008)) have been represented by probability distributions, possibility distributions, hybrid numbers (Kaufmann and Gupta, 1985) or random fuzzy variables (RFVs) (Ferson and Ginzburg, 1995), belief functions of evidence theory (Shafer, 1976). A joint uncertainty propagation process has been used by various authors (Ferson and Ginzburg, 1995, Baudrit et al., 2006, Bárdossy and Fodor, 2004, Baraldi and Zio, 2008) to propagate uncertainties associated to the elementary variables onto the system-level function with the 
least possible loss of information. The propagation process is carried out by the Monte Carlo simulation (MCS) method (Baudrit et al., 2006), which however can be quite time-consuming (Ferson, 1996) and can have difficulties in handling dependencies (Baudrit et al., 2006). For analytical joint uncertainty propagation, the hybrid arithmetic approach based on RFVs has been proposed (Cooper et al., 1996, Ferson and Ginzburg, 1995), assuming the orthogonality between aleatory and epistemic uncertainty and mixing the probabilistic convolution for aleatory uncertainty and the fuzzy calculus for epistemic uncertainty. It is recalled that a hybrid number is a random distribution of fuzzy numbers (i.e. RFV) and also is a fuzzy probability distribution (i.e. fuzzy random variable): these concepts are interchangeable since they lead to equivalent representations, and complementary interpretations and calculation strategies (Ferson and Ginzburg, 1995). In the rest of this paper, we use the term RFV in line with the existing studies.

In this work, we will draw the theoretical connection between UGF and RFV to define a new UGF, hybrid UGF (HUGF), which extends the conventional UGF to represent the RFV whose random dimension is discrete for the multi-state case. Algebraic operators on HUGF will be introduced for joint uncertainty propagation and an efficient algorithm will be developed to extract probability boxes ( $p$ boxes) of system availability from the system HUGF.

The rest of the paper is organized as follows. Section 2 illustrates, through a multi-state model of solar generation, the co-existence of aleatory and epistemic uncertainty in MSS and presents the assumptions made for MSS modeling. In Section 3, the concept of RFV is recalled and HUGF is proposed as theoretical extension of UGF for RFV representation. In Section 4, the algebraic operators of HUGF are defined. In Section 5, the algorithm extracting the probability boxes ( $p$-boxes) of MSS reliability is proposed. Section 6 presents one case study. Section 7 concludes this work.

\section{MSS WITH ALEATORY AND EPISTEMIC UNCERTAINTIES}

We take the solar generator model from ( $\mathrm{Li}$ and Zio, 2012a) as an illustrative example of multi-state component affected by the two types of uncertainties. Its description is based on two random variables (RVs), solar irradiation and mechanical condition, a set of generation parameters and an energy conversion function (which transfers the irradiation to power output). In practice, there is usually sufficient historical data to capture the variabilities in solar irradiation and mechanical condition. In multi-state setting, solar irradiation $r_{S}$ is discretized into several exclusive states ranging from zero to maximum irradiations; the mechanical condition $m_{S}$ is assumed to be a binary RV taking values $\{0,1\}$, where ' 0 ' means complete failure and ' 1 ' means perfect functioning. The power output $G_{S}$ of one solar generator is given by the following functions:

$$
\begin{aligned}
& G_{S}=g_{S}\left(r_{S}, \boldsymbol{\theta}_{S}, m_{S}\right)=N \cdot I_{y} \cdot V_{y} \cdot F F \cdot m_{S} \\
& I_{y}=r_{S} \cdot\left[I_{s c}+k_{c}\left(T_{c}-25\right)\right] \\
& V_{y}=V_{o c}-k_{v} \cdot T_{c} \\
& T_{c}=T_{a}+r_{S} \cdot \frac{N_{o t}-20}{0.8} \\
& F F=\frac{V_{M P P} I_{M P P}}{V_{o c} \cdot I_{s c}}
\end{aligned}
$$

where $g_{S}(\cdot)$ is the solar energy conversion function, $\quad \boldsymbol{\theta}_{S}=\left(I_{M P P}, I_{S c}, k_{c}, k_{v}, N_{o t}, T_{a}, V_{M P P}, V_{o c}\right)$ is the vector of operation parameters, $N$ is the total number of solar cells in the solar generator, $F F$ is the fill factor, $I_{S c}$ is the short circuit current in A, $k_{c}$ is the current temperature coefficient $\mathrm{A} /{ }^{\circ} \mathrm{C}, T_{c}$ is the cell temperature in ${ }^{\circ} \mathrm{C}, V_{o c}$ is the open-circuit voltage in $\mathrm{V}, k_{v}$ is the voltage temperature coefficient $\mathrm{V} /{ }^{\circ} \mathrm{C}, T_{a}$ is the ambient temperature in ${ }^{\circ} \mathrm{C}$, $N_{o t}$ is the nominal operating temperature in ${ }^{\circ} \mathrm{C}$, $V_{M P P}$ is the voltage at maximum power point in $\mathrm{V}$, and $I_{M P P}$ is the current at maximum power point in A.

In literature, the operation parameters are typically treated as constants. In practice, they often change during the operation phase due to degradation of materials, changes in the operating environment, etc (Giannakoudis et al., 2010). However, often insufficient information is available to model them as RVs, due to the unwillingness of the manufacturers to disclose the commercially sensitive data ( $\mathrm{Li}$ and Zio, 2012b). In this situation, the FVs are one promising alternative. It can be seen from eq. (1) that each realization of $G_{S}$ is a fuzzy number. Essentially, $G_{S}$ is a RFV, which we will show in Sections 3 and 4.

Based on the example above, the following assumptions are made for our MSS modeling:

- For any component $i$, it has $M_{i}+1$ different states $\left\{0,1, \ldots, M_{i}\right\}$ where state $M_{i}$ and 0 are the perfect functioning and the complete failure states, respectively. The generic intermediate state $j$ $\left(0<j<M_{i}\right)$ is a degradation state where the component is partially functioning. The state in$\operatorname{dex} j$ is a crisp value.

- In the model of a component $i, \mathrm{FVs}$ are used to represent model parameters if they are tainted with imprecision. 
- Following assumption 2, the performance of a component $i$ is a discrete RV $G_{i}$ if there is sufficient data to eliminate all the imprecision in its parameters; otherwise it will be a RFV $\ddot{G}_{i}$ (or a pure FV $\widetilde{G}_{i}$, if only FVs are involved in the component model).

- The state of the system is completely determined by the state of its components.

\section{HUGF FOR HYBRID UNCERTAINTY REPRESENTATION IN MSS}

In this Section, the definition of RFV is first recalled. Then the UGF representation of RFV, named HUGF, is formally defined.

\section{$3.1 R F V$}

RFV was first introduced by Kaufmann and Gupta (Kaufmann and Gupta, 1985) as a tool to jointly express the epistemic and aleatory uncertainties. Later, RFV were extended by Cooper et al. (Cooper et al., 1996) and Baudrit et al. (Baudrit et al., 2006) for hybrid uncertainty propagation in the area of risk analysis. Given the monotonicity of cumulative distribution functions (CDFs) of RVs and the nestedness of possbility distribution functions of FVs, the formal definition of RFV proposed by Ferson and Ginzburg (Ferson and Ginzburg, 1995) is presented as follows.

Definition 1 (Ferson and Ginzburg, 1995) Let $\boldsymbol{F}$ denote the set of all CDFs defined on the real number set $\mathfrak{R}$ and each element $F \in \boldsymbol{F}$ is an onto function $F: \Re \rightarrow[0,1]$ such that $F\left(x_{1}\right) \geq F\left(x_{2}\right)$ whenever $x_{1}>x_{2}$. A RFV is a set of closed intervals, each characterized by a pair of functions from $\boldsymbol{F}$ :

$$
H:[0,1] \rightarrow \boldsymbol{F} \times \boldsymbol{F}: \alpha \mapsto\left[\underline{F}_{\alpha}, \bar{F}_{\alpha}\right]
$$

such as for $\alpha_{1}, \alpha_{2} \in[0,1], \quad \bar{F}_{\alpha_{1}}(x) \geq \bar{F}_{\alpha_{2}}(x) \geq$ $\underline{F}_{\alpha_{2}}(x) \geq \underline{F}_{\alpha_{1}}(x)$ wherenever $\alpha_{1}<\alpha_{2}$, where $\alpha_{1}$ and $\alpha_{2}$ represent fuzzy membership values of $x$.

Example: Figure 1a depicts the three-dimension representation of a RFV. The $x$-axis is the real number line, the $F$-axis has the cumulative probability values, and the $\pi$-axis contains the possibility values. The shaded area at $\alpha \in(0,1)$ level includes all the closed probability intervals characterized by $\underline{F}_{\alpha}$ as the lower bound and $\bar{F}_{\alpha}$ as the upper bound. Figure 1c shows the two-dimension representation of this RFV and its $\alpha$ level probability intervals. Figure $1 b$ depicts the intersection of the RFV with the plan $F(x)=p$; similarly, Figure 1d depicts this intersection in the two-dimension representation.
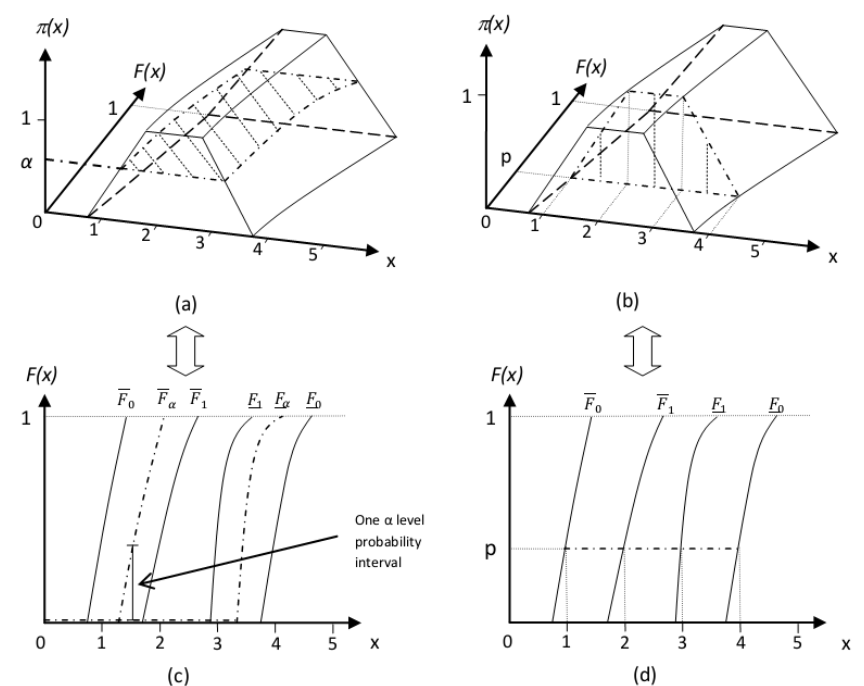

Figure 1.Three-dimension and two-dimension representations of an example RFV

\subsection{HUGF representation of $R F V$}

The UGF for a discrete RV $X$ is defined as:

$$
u_{X}(z)=\sum_{j=0}^{J} p_{j} z^{x_{j}}
$$

where $z$ is the base of the z-transform, $J+1$ is the sample space size of $X, x_{j}$ is the $j$-th sample of $X$, and $p_{j}$ is the probability mass attached to $x_{j}$ satisfying $\sum_{j=0}^{J} p_{j}=1$. The u-function is useful in representing the PMF of discrete RV because it preserves some basic properties of the momentgenerating function, which uniquely determines its PMF (Lisnianski and Levitin, 2003).

Besides Definition 1, RFV can also be regarded as a random distribution of fuzzy numbers (Cooper et al., 1996). In the context of MSS, the random distribution is defined on a finite set of elements, e.g. crisp numbers or fuzzy numbers. Figure 2 shows such a RFV.

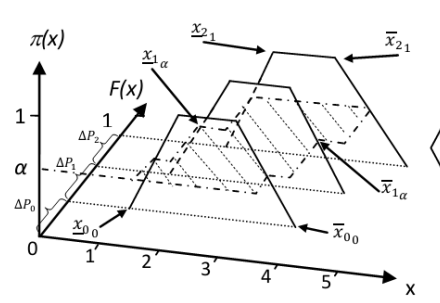

(a)

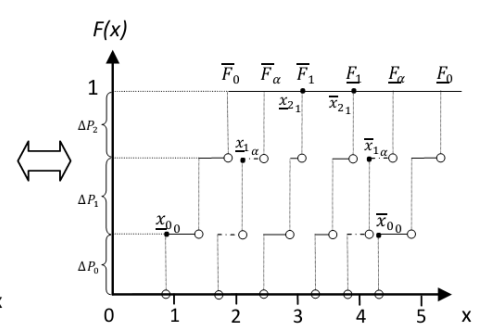

(b)
Figure 2. An example RFV defined on finite fuzzy numbers

Definition 2. For a RFV $\ddot{X}$ defined on a finite set of fuzzy numbers $\boldsymbol{\pi},|\boldsymbol{\pi}|=J+1$, its u-function, denoted by $u_{\ddot{X}}(z)$, is written as follows: 


$$
u_{\ddot{X}}(z)=\sum_{j=0}^{J} p_{j} z^{\tilde{X}_{j}}=\sum_{j=0}^{J} p_{j} z^{\left[\underline{\underline{x}}_{\alpha_{\alpha}}, \bar{x}_{j}\right]}
$$

It is noted that this definition satisfies the basic property of UGF: the coefficient and exponent are not necessarily scalar variables but can be other mathematical objects (i.e. FV) (Lisnianski and Levitin, 2003). It is seen that (3) is the special case of (4). On the other hand, if there is only one term of $\mathrm{z}$, with its coefficient equal to 1 , then (4) will reduce to the following expression,

$$
u_{\tilde{X}}(z)=z^{\tilde{X}}=z^{\left[\underline{x_{\alpha}}, \bar{x}_{\alpha}\right]}
$$

which is the u-function of a pure FV. Recall that $\pi_{\tilde{X}}(x)$ can be uniquely determined by its $\alpha$-cut set: $\left[\underline{x}_{\alpha}, \bar{x}_{\alpha}\right]$, thus (4) defines a one-to-one correspondence to $\tilde{X}$.

\section{JOINT UNCERTAINTY PROPAGATION IN MSS}

This Section defines the HUGF composition operators to combine different types of uncertain variables. Based on the HUGF composition operators, the method for joint uncertainty propagation in MSS reliability assessment is then proposed.

\subsection{HUGF composition operator for joint uncertainty propagation}

Because RFV treats the two types of uncertainties separately, the composition operator of HUGF has to combine the properties of both probabilistic UGF composition operator (Ushakov, 1986) and fuzzy extension principle (Dubois et al., 2000). In the following three cases, we show that the conventional UGF composition operator $\otimes_{f}$ is applicable on HUGF compositions if its structure function $f(\cdot)$ supports fuzzy arithmetic operations.

Case 1: $\otimes_{f}$ between the u-functions of two FVs $\tilde{X}_{1}$ and $\tilde{X}_{2}$,

$$
u_{\tilde{X}_{1}}(z) \otimes_{f} u_{\tilde{X}_{2}}(z)=z^{f\left(\tilde{X}_{1}, \tilde{X}_{2}\right)}
$$

The extension principle (Dubois et al., 2000) reads that $\pi_{\tilde{Y}}(y)=\sup _{y=f\left(x_{1}, x_{2}\right)} \min \left(\pi_{\tilde{X}_{1}}\left(x_{1}\right), \pi_{\tilde{X}_{2}}\left(x_{2}\right)\right)$. For example, in the denominator of eq. (1.e) if we have $\tilde{I}_{s c}=[1+\alpha, 4-\alpha]$ and $\tilde{V}_{o c}=[2+\alpha, 4-$ $\alpha$ ], then u-function of the denominator can be written as

$$
u_{\tilde{I}_{s c}}(z) \bigotimes_{\times} u_{\widetilde{V}_{o c}}(z)=z^{\tilde{I}_{s c} \times \widetilde{V}_{o c}}=z^{[(1+\alpha) \times(2+\alpha),(4-\alpha) \times(4-\alpha)]}
$$

It is noted that fuzzy arithmetic assumes the total dependence between the $\alpha$-cuts (Baudrit et al., 2006).

Case 2: $\otimes_{f}$ between one $R V X_{1}$ and one $F V \tilde{X}_{2}$,

$$
u_{X_{1}}(z) \bigotimes_{f} u_{\tilde{X}_{2}}(z)=\sum_{j_{1}=0}^{J_{1}} p_{1 j_{1}} z^{f\left(x_{1 j_{1}}, \tilde{X}_{2}\right)}
$$

For example, on the right hand side of eq. (1.b) the first term is $r_{S} \cdot \tilde{I}_{s c}$. Suppose that $r_{S}$ has three state levels $(0,0.2,0.8)$ with the probability vector $(0.4$, $0.4,0.2)$; then, the u-function of this term can be written as

$$
\begin{aligned}
u_{r_{S}}(z) \otimes_{\times} u_{\tilde{I}_{S C}}(z) & \\
= & 0.4 z^{0}+0.4 z^{[0.2(1+\alpha), 0.2(4-\alpha)]} \\
+ & 0.2 z^{[0.8(1+\alpha), 0.8(4-\alpha)]}
\end{aligned}
$$

Case 3: $\otimes_{f}$ between two random FVs $\ddot{X}_{1}$ and $\ddot{X}_{2}$,

$$
u_{\ddot{X}_{1}}(z) \otimes_{f} u_{\ddot{X}_{2}}(z)=\sum_{j_{1}=0}^{J_{1}} \sum_{j_{2}=0}^{J_{2}} p_{1 j_{1}} p_{2 j_{2}} z^{f\left(\tilde{X}_{1 j_{1}}, \tilde{X}_{2 j_{2}}\right)}
$$

For example, by substituting eq. (1.d) into eq. (1.b), the first and second terms become $r_{S} \cdot \tilde{I}_{s c}$ and $r_{S} \cdot \tilde{k}_{c} \cdot \tilde{T}_{a}$, respectively. Let $\tilde{k}_{c}=[1+\alpha, 4-\alpha]$ and $\tilde{T}_{a}=[2+\alpha, 5-\alpha]$; then, we have the following $\mathrm{u}$-function for the addition of these two terms

$$
\begin{aligned}
& u_{r_{S} \cdot \tilde{I}_{s c}}(z) \otimes_{+} u_{r_{S} \tilde{k}_{c} \cdot \tilde{T}_{a}}(z) \\
& =\left(0.4 z^{0}+0.4 z^{[0.2(1+\alpha), 0.2(4-\alpha)]}\right. \\
& \left.+0.2 z^{[0.8(1+\alpha), 0.8(4-\alpha)]}\right) \otimes_{+}\left(0.4 z^{0}\right. \\
& +0.4 z^{[0.2(1+\alpha)(2+\alpha), 0.2(4-\alpha)(5-\alpha)]} \\
& +0.2 z^{[0.8(1+\alpha)(2+\alpha), 0.8(4-\alpha)(5-\alpha)])} \\
& =0.16 z^{0}+0.16 z^{[0.2(1+\alpha)(2+\alpha), 0.2(4-\alpha)(5-\alpha)]} \\
& +0.08 z^{[0.8(1+\alpha)(2+\alpha), 0.8(4-\alpha)(5-\alpha)]} \\
& +0.16 z^{[0.2(1+\alpha), 0.2(4-\alpha)]} \\
& +0.16 z^{[0.2(1+\alpha)(3+\alpha), 0.2(4-\alpha)(6-\alpha)]} \\
& +0.08 z^{[0.2(1+\alpha)(9+4 \alpha), 0.2(4-\alpha)(21-4 \alpha)]} \\
& +0.08 z^{[0.8(1+\alpha), 0.8(4-\alpha)]} \\
& +0.08 z^{[0.2(1+\alpha)(6+\alpha), 0.2(4-\alpha)(9-\alpha)]} \\
& +0.04 z^{[0.8(1+\alpha)(3+\alpha), 0.8(4-\alpha)(6-\alpha)]}
\end{aligned}
$$

Note that all the FVs and RVs used in the three cases above are artificially created for illustration purposes, aiming at showing the capability of the HUGF composition operator of handling the combination of variables with different uncertainty representations.

In general, the HUGF composition operator of $N$ u-functions, i.e. uncertain variables, is defined as follows 


$$
\begin{aligned}
& \bigotimes_{f}\left(u_{\ddot{X}_{1}}(z), u_{\ddot{X}_{2}}(z), \ldots, u_{\ddot{X}_{N}}(z)\right) \\
& =\sum_{j_{1}=0}^{J_{1}} \ldots \sum_{j_{N}=0}^{J_{N}} \prod_{k=1}^{N} p_{k j_{i}} z^{f\left(\tilde{X}_{1 j_{1}}, \tilde{X}_{2 j_{1}}, \ldots, \tilde{X}_{N j_{N}}\right)}
\end{aligned}
$$

It is noted that for the case of two arguments, the following two notations are interchangeable:

$$
\bigotimes_{f}\left(u_{\ddot{X}_{1}}(z), u_{\ddot{X}_{2}}(z)\right)=u_{\ddot{X}_{1}}(z) \otimes_{f} u_{\ddot{X}_{2}}(z)
$$

Two basic properties of $\otimes_{f}$, namely the associative and communicative properties, are recalled for the reduction of composition computation time. If the function $f(\cdot)$ possesses the associative property for any of its variables, then $\otimes_{f}$ also possesses this property

$$
\begin{aligned}
& \otimes_{f}\left(u_{\ddot{X}_{1}}(z), \ldots, u_{\ddot{X}_{k}}(z), u_{\ddot{X}_{k+1}}(z), \ldots, u_{\ddot{X}_{N}}(z)\right)= \\
& \quad \otimes_{f}\left(\otimes_{f}\left(u_{\ddot{X}_{1}}(z), \ldots, u_{\ddot{X}_{k}}(z)\right), \otimes_{f}\left(u_{\ddot{X}_{k+1}}(z), \ldots, u_{\ddot{X}_{N}}(z)\right)\right)
\end{aligned}
$$

If the function $f(\cdot)$ possesses the communicative property for any of its variables, then $\otimes_{f}$ also possesses this property

$$
\begin{aligned}
\otimes_{f}\left(u_{\ddot{X}_{1}}(z), \ldots, u_{\ddot{X}_{k}}(z), u_{\ddot{X}_{k+1}}(z), \ldots, u_{\ddot{X}_{N}}(z)\right)= \\
\otimes_{f}\left(u_{\ddot{X}_{1}}(z), \ldots, u_{\ddot{X}_{k+1}}(z), u_{\ddot{X}_{k}}(z), \ldots, u_{\ddot{X}_{N}}(z)\right)
\end{aligned}
$$

By applying these two properties, the elementary RVs and FVs might be eventually separated:

$$
\begin{aligned}
& \otimes_{f}\left(u_{X_{1}}(z), \ldots, u_{\tilde{X}_{N}}(z)\right)= \\
& \quad \otimes_{f}\left(\bigotimes_{f}\left(u_{X_{1}}(z), \ldots, u_{X_{k}}(z)\right), \otimes_{f}\left(u_{\tilde{X}_{k+1}}(z), \ldots, u_{\tilde{X}_{N}}(z)\right)\right)
\end{aligned}
$$

In this way, the u-functions of FVs can be processed prior to the combination with the u-function of RVs, which involves multiplication to the polynomials.

Let $\ddot{D}=\ddot{G}_{S}-w$ where $w$ is the arbitrary demand, the procedures of computing the MSS HUGF are presented as follows:

(1) Build the u-function for each component. For component $i$ affected by both types of uncertainties, obtain $u_{\ddot{G}_{i}}(z)$ by combining the elementary FVs or RVs using $\otimes_{f}$ with the consideration of the communicative and associative rules;

(2) Obtain the HUGF $u_{\ddot{G}_{S Y S}}(z)$ using $\otimes_{f}$ to combine the component u-functions according to the system structure function $\ddot{G}_{S Y S}=$ $\varphi\left(\ddot{G}_{1}, \ldots, \ddot{G}_{n}\right)$, where the communicative and associative rules also apply;
(3) Compute the system HUGF, $u_{\ddot{D}}(z)=$ $u_{\ddot{G}_{S Y S}}(z) \otimes_{-} u_{w}(z)$.

This method involves both the fuzzy arithmetic and probabilistic convolution operations, either of which could lead to high computational cost. To reduce the computational complexity of this method, approximation techniques have to be applied especially when the MSS contains a large number of uncertain variables.

\section{EXTRACTING INFORMATION FROM SYSTEM HUGF}

As described in Section $4, \ddot{D}$ is represented by a RFV. Thus, the MSS availability $A(w)=\operatorname{Pr}(\ddot{D} \geq$ $0)=1-\operatorname{Pr}(\ddot{D}<0)$ is no longer a precise value but a set of probability intervals, one for each $\alpha$ level. This complete information is, however, too complex to be utilized by the decision maker. In order to extract useful information from these probability intervals, the post-treatment methods have been proposed. In this Section, we present two of them: $p$-boxes (Ferson and Ginzburg, 1996) and homogenous post-processing (Baudrit et al., 2006), and propose one algorithm to produce them from the system HUGF.

\section{1 p-boxes}

The concept of $p$-box is similar to that of RFV. (Ferson and Ginzburg, 1996) proposed to fix the $\alpha$ level and, then, build the lower and upper probability bounds $\left[\underline{F}_{\alpha}(B), \bar{F}_{\alpha}(B)\right]$ of an event $B$, i.e. $\ddot{D}<0$. Two representative cases of the $p$-boxes are $\alpha=0$ and $\alpha=1$. The $p$-box $\left[\underline{F}_{0}(B), \bar{F}_{0}(B)\right]$ corresponds to a pessimistic condition where the imprecision is maximized while the $p$-box $\left[\underline{F}_{1}(B), \bar{F}_{1}(B)\right]$ corresponds to an optimistic situation where the imprecision is minimized. It is noted that even in the optimistic case, there still can be imprecision if the $\alpha=1$ level of each FV is not a single number.

\subsection{Homogenous post-processing}

(Baudrit et al., 2006) proposed this method to extract only one lower and one upper probability bounds, which take the fuzzy mean (Dubois and Prade, 1987) over all $p$-boxes:

$$
\underline{F}_{a v}(B)=\int_{0}^{1} \underline{F}_{\alpha}(B) d \alpha \text { and } \bar{F}_{a v}(B)=\int_{0}^{1} \bar{F}_{\alpha}(B) d \alpha
$$


It can be shown that $\forall B \subseteq \Re, F_{0}(B) \leq$ $\underline{F}_{a v}(B) \leq F_{1}(B)$ and $\bar{F}_{1}(B) \leq \bar{F}_{a v}(B) \leq \bar{F}_{0}(B)$. (Baudrit et al., 2006), then, established the link between the interval $\left[F_{a v}(B), \bar{F}_{a v}(B)\right]$ and the belief functions of evidence theory, under the condition that there are finite elements in the probability sample and possibility sample spaces, which is not true in our case. Figure 4 depicts the CDF curves of the $p$-boxes at the $\alpha$ levels equal to 0 and 1 , and the average $p$-boxes.

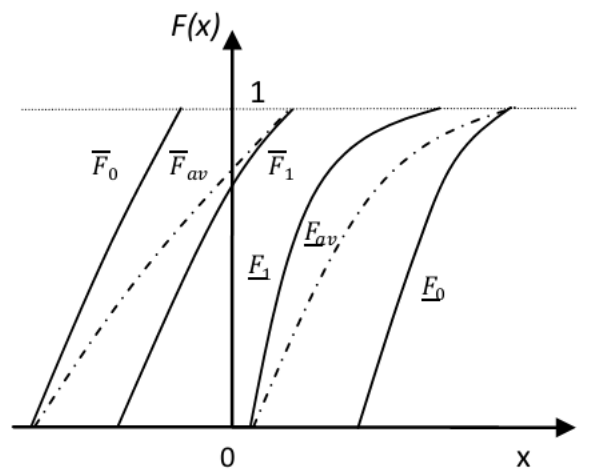

Figure 4. CDF curves of $\left[\underline{F}_{0}(x), \bar{F}_{0}(x)\right],\left[\underline{F}_{a v}(x), \bar{F}_{a v}(x)\right]$, and $\left[\underline{F}_{1}(x), \bar{F}_{1}(x)\right]$

\subsection{Algorithm for the system availability p-boxes extraction}

Let $B$ denote the event $\ddot{D}<0$; we have the system availability $p$-box: $\left[\underline{A}_{\alpha}, \bar{A}_{\alpha}\right]$ where $\underline{A}_{\alpha}=1-$ $\bar{F}_{\alpha}(B)$ and $\bar{A}_{\alpha}=1-\underline{F}_{\alpha}(B)$. To show the extraction of $\left[\underline{A}_{\alpha}, \bar{A}_{\alpha}\right]$ (at a fixed $\alpha$ level), we take $\underline{A}_{\alpha}$ as an example. By definition of $\bar{F}_{\alpha}(B)$ we have $\underline{A}_{\alpha}=\sum_{\underline{d}_{j} \geq 0} p\left(\underline{d}_{j_{\alpha}}\right)$, where $j=0, \ldots, J_{\ddot{D}}$ and $J_{\ddot{D}}$ is the highest state of $\ddot{D}$. Its computation is straightforward and $\bar{A}_{\alpha}$ can be calculated similarly. To show the extraction of the average availability $p$-box $\left[\underline{A}_{a v}, \bar{A}_{a v}\right]$, we take $\underline{A}_{a v}$ as an example. By definition we have $\underline{A}_{a v}=\int_{0}^{1} \sum_{\underline{d}_{j_{\alpha}} \geq 0} p\left(\underline{d}_{j_{\alpha}}\right) d \alpha$. For its computation, at a particular state $j$ the following three exclusive conditions are identified: 1) $\underline{d}_{j_{\alpha}} \geq 0$ for any $\alpha \in[0,1]$, then we have $\int_{0}^{1} p\left(\underline{d}_{j_{\alpha}}\right) d \alpha=$ $p\left(\underline{d}_{j_{\alpha}}\right)$ because $p\left(\underline{d}_{j}\right)$ is a constant for any $\left.\alpha ; 2\right)$ $\underline{d}_{j_{\alpha}}<0$ for any $\alpha \in[0,1]$, then we have $\int_{0}^{1} p\left(\underline{d}_{j_{\alpha}}\right) d \alpha=0$; 3) $\underline{d}_{\alpha_{\alpha_{1}}}<0$ and $\underline{d}_{\alpha_{2}} \geq 0$ for certain $\alpha_{1}, \alpha_{2} \in[0,1]$ and $\alpha_{1}<\alpha_{2}$, then we have $\int_{0}^{1} p\left(\underline{d}_{j}\right) d \alpha=\left(1-\alpha_{L}^{*}\right) \times p\left(\underline{d}_{j_{\alpha}}\right)$

where $\underline{d}_{\alpha_{L}^{*}}=0$ (See Fig. 5). $\bar{A}_{a v}$ can be obtained similarly.

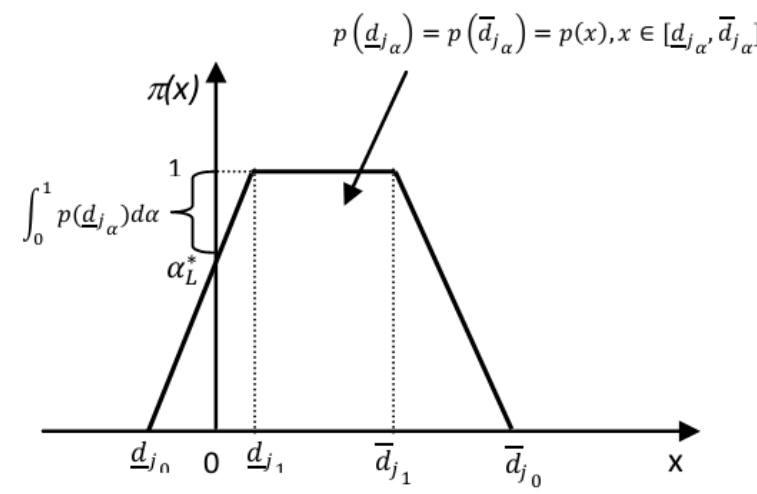

Figure 5. For a particular state $j$, the computation of $\int_{0}^{1} p\left(\underline{d}_{j_{\alpha}}\right) d \alpha$ when $\underline{d}_{\alpha_{1}}<0$ and $\underline{d}_{\alpha_{2}} \geq 0$ for certain $\alpha_{1}, \alpha_{2} \in[0,1]$ and $\alpha_{1}<\alpha_{2}$

Based upon the discussions above, the following algorithm is designed for the $p$-boxes extraction:

Initialize: set $\underline{A}_{\alpha}=\bar{A}_{\alpha}=\underline{A}_{a v}=\bar{A}_{a v}=0$

For $j=0$ to $J_{\ddot{D}}$ do

Obtain $\underline{x}_{j}$ and $\bar{x}_{j_{\alpha}}$ by substituting the given $\alpha$ value into the fuzzy number expression.

If $\underline{d}_{j_{\alpha}} \geq 0$, then $\underline{A}_{\alpha} \leftarrow \underline{A}_{\alpha}+p_{j}$.

If $\bar{d}_{j_{\alpha}} \geq 0$, then $\bar{A}_{\alpha} \leftarrow \bar{A}_{\alpha}+p_{j}$.

If $\underline{d}_{j_{0}} \geq 0$, then $\underline{A}_{a v} \leftarrow \underline{A}_{a v}+p_{j}$;

Else-if $\underline{d}_{j_{0}}<0$ and $\underline{d}_{j_{1}} \geq 0$, then calculate $\alpha_{L}^{*}$ and $\underline{A}_{a v} \leftarrow \underline{A}_{a v}+p_{j} \times\left(1-\alpha_{L}^{*}\right)$.

If $\bar{d}_{j_{1}} \geq 0$, then $\bar{A}_{a v} \leftarrow \bar{A}_{a v}+p_{j}$;

Else-if $\bar{d}_{j_{1}}<0$ and $\bar{d}_{j_{0}} \geq 0$, then calculate $\alpha_{U}^{*}$ and End $\bar{A}_{a v} \leftarrow \bar{A}_{a v}+p_{j} \times \alpha_{U}^{*}$.

\section{CASE STUDY}

In this Section, we demonstrate the proposed HUGF method on the three-element flow transmission system, whose block diagram is shown in Fig. 6.

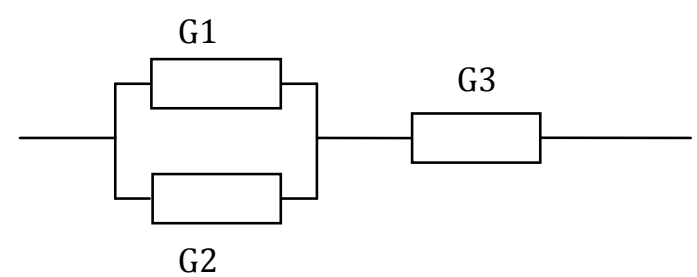

Figure 6. A three component flow transmission system

The u-function of each component performance variable is presented as follows,

$$
\begin{aligned}
& u_{G_{1}}(z)=0.1 z^{1}+0.4 z^{3}+0.3 z^{4}+0.2 z^{5} \\
& u_{\ddot{G}_{2}}(z)=0.1 z^{[\alpha, 3-\alpha]}+0.5 z^{[2+\alpha, 4-\alpha]}+0.4 z^{[3+\alpha, 5-\alpha]} \\
& u_{\ddot{G}_{3}}(z)=0.3 z^{[6+\alpha, 8-\alpha]}+0.3 z^{[7+\alpha, 9-\alpha]}+0.4 z^{[8+\alpha, 10-\alpha]}
\end{aligned}
$$

Then, HUGF of the system can be written as: 


$$
\begin{aligned}
& u_{S \ddot{Y} S}(z)=\left(u_{G_{1}}(z) \otimes_{+} u_{\ddot{G}_{2}}(z)\right) \otimes_{\min } u_{\ddot{G}_{3}}(z) \\
& =\left(0.01 z^{[1+\alpha, 4-\alpha]}+0.05 z^{[3+\alpha, 5-\alpha]}+0.04 z^{[3+\alpha, 6-\alpha]}\right. \\
& +0.04 z^{[4+\alpha, 6-\alpha]}+0.03 z^{[4+\alpha, 7-\alpha]} \\
& +0.2 z^{[5+\alpha, 7-\alpha]}+0.02 z^{[5+\alpha, 8-\alpha]} \\
& +0.31 z^{[6+\alpha, 8-\alpha]}+0.22 z^{[7+\alpha, 9-\alpha]} \\
& \left.+0.08 z^{[8+\alpha, 10-\alpha]}\right) \otimes_{\min }\left(0.3 z^{[6+\alpha, 8-\alpha]}\right. \\
& \left.+0.3 z^{[7+\alpha, 9-\alpha]}+0.4 z^{[8+\alpha, 10-\alpha]}\right) \\
& =0.01 z^{[1+\alpha, 4-\alpha]}+0.05 z^{[3+\alpha, 5-\alpha]}+0.04 z^{[3+\alpha, 6-\alpha]} \\
& +0.04 z^{[4+\alpha, 6-\alpha]}+0.03 z^{[4+\alpha, 7-\alpha]} \\
& +0.2 z^{[5+\alpha, 7-\alpha]}+0.02 z^{[5+\alpha, 8-\alpha]} \\
& +0.4 z^{[6+\alpha, 8-\alpha]}+0.178 z^{[7+\alpha, 9-\alpha]} \\
& +0.032 z^{[8+\alpha, 10-\alpha]}
\end{aligned}
$$

Suppose that the load demand is a constant value with 4.25 (in arbitrary units), then the system HUGF is:

$$
\begin{aligned}
& u_{\ddot{D}}(z)=0.01 z^{[-3.25+\alpha,-0.25-\alpha]}+0.05 z^{[-1.25+\alpha, 0.75-\alpha]} \\
& +0.04 z^{[-1.25+\alpha, 1.75-\alpha]} \\
& +0.04 z^{[-0.25+\alpha, 1.75-\alpha]} \\
& +0.03 z^{[-0.25+\alpha, 2.75-\alpha]}+0.2 z^{[0.75+\alpha, 2.75-\alpha]} \\
& +0.02 z^{[0.75+\alpha, 3.75-\alpha]}+0.4 z^{[1.75+\alpha, 3.75-\alpha]} \\
& +0.178 Z^{[2.75+\alpha, 4.75-\alpha]} \\
& +0.032 z^{[3.75+\alpha, 5.75-\alpha]}
\end{aligned}
$$

Based on this u-function, the useful quantities for $p$-boxes constructions are presented in Table 1 .

Table 1. Quantities (in arbitrary units) for constructing $p$ boxes

\begin{tabular}{|c|c|c|c|c|c|c|l|}
\hline Term & $\underline{d}_{j_{0}}$ & $\bar{d}_{j_{0}}$ & $\underline{d}_{j_{1}}$ & $\bar{d}_{j_{1}}$ & $\alpha_{L}^{*}$ & $\alpha_{U}^{*}$ & Probability \\
\hline 1 & -3.25 & -0.25 & -2.25 & -1.25 & & & 0.01 \\
\hline 2 & -1.25 & 0.75 & -0.25 & -0.25 & & 0.75 & 0.05 \\
\hline 3 & -1.25 & 1.75 & -0.25 & 0.75 & & & 0.04 \\
\hline 4 & -0.25 & 1.75 & 0.75 & 0.75 & 0.25 & & 0.04 \\
\hline 5 & -0.25 & 2.75 & 0.75 & 1.75 & 0.25 & & 0.03 \\
\hline 6 & 0.75 & 2.75 & 1.75 & 1.75 & & & 0.2 \\
\hline 7 & 0.75 & 3.75 & 1.75 & 2.75 & & & 0.02 \\
\hline 8 & 1.75 & 3.75 & 2.75 & 2.75 & & & 0.4 \\
\hline 9 & 2.75 & 4.75 & 3.75 & 3.75 & & & 0.178 \\
\hline 10 & 3.75 & 5.75 & 4.75 & 4.75 & & & 0.032 \\
\hline
\end{tabular}

According to our algorithm, the upper and lower bounds of system availability $p$-boxes are computed as follows:

$$
\begin{gathered}
\underline{A}_{0}=0.2+0.02+0.4+0.178+0.032=0.83 \\
\overline{\bar{A}}_{0}=0.05+0.04+0.04+0.03+0.2+0.02+0.4+0.178 \\
\quad+0.032=0.99 \\
A_{1}=0.04+0.03+0.2+0.02+0.4+0.178+0.032=0.9 \\
\overline{\bar{A}}_{1}=0.04+0.04+0.03+0.2+0.02+0.4+0.178 \\
\quad+0.032=0.94 \\
\underline{A}_{a v}=0.75 \times 0.04+0.75 \times 0.03+0.2+0.02+0.4+0.178 \\
\quad+0.032=0.8825 \\
\bar{A}_{a v}=0.75 \times 0.05+0.04+0.04+0.03+0.2+0.02+0.4 \\
\quad+0.178+0.032=0.9775
\end{gathered}
$$

Therefore, $\left[\underline{A}_{0}, \bar{A}_{0}\right]=[0.83,0.99],\left[\underline{A}_{a v}, \bar{A}_{a v}\right]=$ $[0.8825,0.9775]$, and $\left[\underline{A}_{1}, \bar{A}_{1}\right]=[0.9,0.94]$.

\section{CONCLUSIONS}

Aleatory and epistemic uncertainties always coexist in the models for the assessment of industrial systems. How to properly handle them poses challenges to the reliability engineers. In this work, we have proposed an efficient approach based on UGF for joint uncertainty representation, propagation and exploitation in reliability assessments of MSS. Drawing from the well-established RFV theory, HUGF has shown to be adequate for the representation of RFVs defined on a finite set of FVs. Based upon this foundation, the composition operator of HUGF has been defined by combining probabilistic convolution with the fuzzy extension principle. Finally, an efficient algorithm has been designed to extract reliability $p$-boxes from the system HUGF.

\section{REFERENCES}

Apostolakis, G. E. 1990. The concept of probability in safety assessments of technological systems. Science, 250(4986), 1359-1364.

Baraldi, P., and E. Zio 2008. A combined Monte Carlo and possibilistic approach to uncertainty propagation in event tree analysis. Risk Analysis, 28(5), 1309-1325.

Bárdossy, G., and J. Fodor 2004. Evaluation of uncertainties and risks in geology: new mathematical approaches for their handling: Springer.

Baudrit, C., D. Dubois, and D. Guyonnet 2006. Joint propagation of probabilistic and possibilistic information in risk assessment. IEEE Transactions on Fuzzy Systems, 14(5), 593608.

Cai, K. Y. 1996. Introduction to Fuzzy Reliability: Kluwer Academic Pub-lishers.

Chen, S. M. 1994. Fuzzy system reliability analysis using fuzzy number arithmetic operations. Fuzzy Sets and Systems, 6431-38.

Cooper, J. A., S. Ferson, and L. Ginzburg 1996. Hybrid processing of stochastic and subjective uncertainty data. Risk Analysis, 16(6), 785-791.

Ding, Y., M. J. Zuo, A. Lisnianski, and W. Li 2010. A Framework for Reliability Approximation of Multi-State Weighted k-out-of-n Systems. Reliability, IEEE Transactions on, 59(2), 297-308.

Ding, Y., M. J. Zuo, A. Lisnianski, and Z. Tian 2008. Fuzzy Multi-State Systems: General Definitions, and Performance Assessment. IEEE Transactions on Reliability, 57(4), 589-594. 
Dubois, D., H. T. Nguyen, and H. Prade 2000. Possibility theory, probability and fuzzy sets: Misunderstandings, bridges and gaps. In: DUBOIS, D. \& PRADE, H. (eds.) Fundamentals of Fuzzy Sets. Boston, MA: Kluwer.

Dubois, D., and H. Prade 1987. The mean value of a fuzzy number. Fuzzy Sets and Systems, 24(3), 279-300.

Ferson, S. 1996. What Monte Carlo methods cannot do. Human and Ecological Risk Assessment: An International Journal, 2(4), 990-1007.

Ferson, S., and L. R. Ginzburg. Hybrid arithmetic. ISUMA-NAFIPS, 1995 Los Alamitos, CA. 619-623.

Ferson, S., and L. R. Ginzburg 1996. Different methods are needed to propagate ignorance and variability. Reliability Engineering \& Systems Safety, 54133-144.

Giannakoudis, G., A. I. Papadopoulos, P. Seferlis, and S. Voutetakis 2010. Optimum design and operation under uncertainty of power systems using renewable energy sources and hydrogen storage. International Journal of Hydrogen Energy, 35872-891.

Helton, J. C. 2004. Alternative Representations of Epistemic Uncertainty. Reliability Engineering \& System Safety, 85(1-3), 1-10.

Kaufmann, A., and M. M. Gupta 1985. Introduction to Fuzzy Arithmetic: Theory and Applications, New York: Van Nostrand Reinhold.

Li, C. Y., X. Chen, X. S. Yi, and J. Y. Tao 2011. Interval-Valued Reliability Analysis of Multi-State Systems. IEEE Transactions on Reliability, 60(1), 323-330.

Li, Y. F., and E. Zio 2012a. A multi-state model for the reliability assessment of a distributed generation system via universal generating function. Reliability Engineering \& System Safety, 10628-36.

Li, Y. F., and E. Zio 2012b. Uncertainty analysis of the adequacy assessment model of a distributed generation system. Renewable Energy, 41235-244.

Lin, C. H., J. C. Ke, and H. I. Huang 2012. Reliability-based measures for a system with an uncertain parameter environment. International Journal of Systems Science, 43(6), 1146-1156.

Lisnianski, A., and G. Levitin 2003. Multi-state System Reliability: Assessment, Optimization and Applications, Singapore: World Scientific.

Natvig, B. 2011. Multistate System Reliability Theory with Applications, New York: Wiley.

Shafer, G. 1976. A Mathematical Theory of Evidence, Princeton, NJ: Princeton Univ. Press.

Singer, D. 1990. A fuzzy set approach to fault tree and reliability analysis. Fuzzy Sets and Systems, 34145-155.
Ushakov, I. 1986. Universal generating function. Soviet Journal of Computer Systems Science, 24(5), 118-129.

Wang, A. S., Y. Luo, G. Y. Tu, and P. Liu 2011. Quantitative Evaluation of HumanReliability Based on Fuzzy-Clonal Selection. IEEE Transactions on Reliability, 60(3), 517-527. 